\title{
Limitations on Transformations from Composite-Order to Prime-Order Groups: The Case of Round-Optimal Blind Signatures
}

\author{
Sarah Meiklejohn ${ }^{1}$, Hovav Shacham ${ }^{1}$, and David Mandell Freeman ${ }^{2}$ \\ 1 University of California, San Diego \\ La Jolla, CA 92037, USA \\ $\{$ smeiklej, hovav\}@cs.ucsd.edu \\ 2 Stanford University \\ Stanford, CA 94305, USA \\ dfreeman@cs.stanford.edu
}

\begin{abstract}
Beginning with the work of Groth and Sahai, there has been much interest in transforming pairing-based schemes in composite-order groups to equivalent ones in prime-order groups. A method for achieving such transformations has recently been proposed by Freeman, who identified two properties of pairings using composite-order groups - "cancelling" and "projecting" — on which many schemes rely, and showed how either of these properties can be obtained using prime-order groups.

In this paper, we give evidence for the existence of limits to such transformations. Specifically, we show that a pairing generated in a natural way from the Decision Linear assumption in prime-order groups can be simultaneously cancelling and projecting only with negligible probability.

As evidence that these properties can be helpful together as well as individually, we present a cryptosystem whose proof of security makes use of a pairing that is both cancelling and projecting. Our example cryptosystem is a simple round-optimal blind signature scheme that is secure in the common reference string model, without random oracles, and based on mild assumptions; it is of independent interest.
\end{abstract}

\section{Introduction}

Composite-order groups were introduced for pairing-based cryptography in 2005 by Boneh, Goh, and Nissim 12 and have since been used to realize a large number of cryptographic systems (see, e.g., the schemes surveyed by Freeman [24]). At the same time, the limited number of elliptic curve families on which compositeorder groups can be instantiated and the larger parameter sizes associated with composite-order groups (cf. 2313]) has motivated research on translating these schemes to or obtaining similar ones in the prime-order setting.

In one of the first papers to unify the composite- and prime-order settings, Groth and Sahai 31 developed non-interactive zero-knowledge schemes that not only can be instantiated either in composite- or prime-order groups, but are 
in fact described identically in either instantiation. What facilitates this flexibility is a new abstraction for pairing-based cryptography in terms of modules over finite commutative rings with an associated bilinear map; this abstraction allows for the simultaneous treatment of three different cryptographic assumptions: the Subgroup Hiding (SGH) assumption of Boneh, Goh, and Nissim [12 in composite-order groups; the Decision Linear (DLIN) assumption of Boneh, Boyen, and Shacham [11, and its $k$-Linear family of generalizations [453] 1 in prime-order groups; and the so-called Symmetric External Diffie-Hellman assumption [7], also in prime-order groups.

More recently, Freeman [24] and Garg, Sahai, and Waters [27] have proposed methods for transforming schemes secure in the composite-order setting into ones secure (under different but analogous assumptions) in the prime-order setting. Freeman, in particular, identifies two properties of pairings on composite-order groups, projecting and cancelling, and shows how either can be obtained in primeorder groups. He then demonstrates how to transform several known cryptosystems that rely on one of these properties from composite- to prime-order groups.

Our contribution: limits on transformations from composite to prime order. In this paper, we show limits to the feasibility of composite-to-prime transformations such as those mentioned above, suggesting that some schemes cannot be transformed mechanically from one setting to the other. In our main theorem, Theorem 6.5. we show that no pairing over prime-order groups can - except in a negligible fraction of cases — be both projecting and cancelling when subgroup indistinguishability relies in a natural way on $k$-Linear, where "natural" simply means that we follow the definition of the assumption as closely as possible.

If no cryptosystem required a pairing that is both projecting and cancelling, however, our Theorem 6.5 would not be particularly interesting. As such, we present a new cryptosystem - a natural pairing-based blind signature scheme that is of independent interest, and discussed below - whose proof of security calls for a pairing that is both projecting and cancelling 2

Blind signatures were introduced by Chaum in 1982 [17. In a blind signature scheme, a user interacts in a protocol with a signer to obtain a signature on a message of the user's choice. When the protocol execution ends, the user obtains the signature but the signer learns nothing about the message that was signed. Blind signatures have been used as a building block in a variety of applications, including electronic cash [20] and electronic voting [19].

One useful feature of a blind signature scheme is concurrency. For example, if a blind signature used to build an electronic cash system does not retain its security even when the signer engages in multiple protocol executions concurrently, it leaves the issuing bank susceptible to denial-of-service attacks. Concurrency turns out to be as difficult to achieve for blind signatures as it is for other cryptographic

\footnotetext{
${ }^{1}$ A family of progressively strictly weaker decisional assumptions, where 1-Linear is DDH and 2-Linear is DLIN.

${ }^{2}$ We emphasize that it is the security proof, not the statement of the scheme, that uses the two pairing properties. We thus do not rule out the possibility that a different proof strategy will show our scheme secure in prime-order groups.
} 
protocols. While many blind signature schemes have proofs of security only for sequential executions of the protocol, the problem is not merely with proofs. In one example, Martinet, Poupard, and Sola 38. show that signatures in a partially blind signature scheme of Cao, Lin and Xue [16] are forgeable if the signer interacts with two users concurrently.

Our contribution: a round-optimal blind signature scheme. As mentioned above, we present a new pairing-based blind signature scheme. Our blind signing protocol is round-optimal: it consists of only two moves (a request and a response), which implies that it is secure even in the presence of concurrent signing protocol executions. Our scheme is practical, has a proof of security (without random oracles) in the common reference string model, and relies for its security on falsifiable and non-interactive assumptions: computational Diffie-Hellman and Subgroup Hiding. These assumptions are milder than those used in any previous practical concurrently secure blind signature, including those in the random oracle model. ("Practical" in this sense means not relying on general NIZKs for NP as a building block.) Our scheme extends in a natural way to give a partially blind signature scheme [3] with the same properties.

Our blind signatures combine the Waters signature scheme [46] with noninteractive witness-indistinguishable proofs developed in a line of papers by Groth, Ostrovsky, and Sahai 30 29 31]. In this structure our scheme is related to the group signature scheme of Boyen and Waters [15. The primary disadvantage of our scheme, as with the Boyen-Waters group signature, is its bit-at-a-time nature, which makes the user's blind signing request large: some 40 kilobytes at the 1024-bit security level. The signer's response and the resulting signatures, however, are short.

Related work. The blind signature literature is extensive and varied. Below, we briefly survey the most closely related schemes with concurrent security; see [54] for more complete recent treatments.

In the random oracle model, there exist elegant round-optimal blind signatures, due to Chaum [18] and Boldyreva [10, that feature short public keys, short signatures, and an efficient blind signing protocol. Unfortunately the security proofs for these schemes rely on strong interactive assumptions: the RSA known-target inversion assumption 9] and the chosen-target CDH assumption (by contrast, the underlying ordinary signatures can be shown secure using RSA and $\mathrm{CDH}$, respectively).

In the common reference string model, several practical concurrently secure blind signature schemes have been proposed. Unlike our scheme, these schemes rely on assumptions that are interactive or whose statement size grows with the number of queries in the reduction (i.e., "q-type"). Kiayias and Zhou 35] give four-move blind and partially-blind signature schemes secure under the (interactive) LRSW assumption [37, the Paillier assumption [42, and DLIN. Okamoto [40] gives four-move blind and partially blind signature schemes based on the (q-type) Two-Variable Strong Diffie-Hellman assumption and Paillier. Fuchsbauer 25] gives two-move blind signature schemes based on the (q-type) 
Asymmetric Double Hidden Strong Diffie-Hellman assumption, the Asymmetric Weak Flexible CDH assumption, and DLIN. Finally, Abe, Haralambiev, and Ohkubo 4 give two-move blind signature schemes based on the ( $q$-type) Simultaneous Flexible Pairing assumption and DLIN. (The last two papers appeared together as [2.)

Also in the common reference string model, blind signatures that use general NIZKs for NP (and are therefore not practical) were given by Juels, Luby, and Ostrovsky 34, Fischlin [22, and Abe and Ohkubo [5]. The Fischlin and AbeOhkubo schemes are round-optimal.

Okamoto [40] first observed that the Waters signature can be combined with witness-indistinguishable proofs for a simple NP language to yield blind and partially blind signatures. Our scheme could be viewed as an instantiation of Okamoto's framework (though we blind the message differently) where we take advantage of Groth-Ostrovsky-Sahai proofs to eliminate a round of interaction.

Until recently, no concurrently secure blind signature schemes were known in the plain public-key model. The first such scheme was given by Hazay et al. [32]; it relies on general NIZKs, and its round complexity is poly-logarithmic in the number of concurrent executions for which security must be guaranteed.

Applications and extensions. Finally, as an application of our techniques, we show (in the full version of our paper 39]) how our blind signatures may be used within the Waters IBE system [46] to yield a blind IBE scheme, as introduced by Green and Hohenberger 28. Compared to Green and Hohenberger's blind extraction protocol, our protocol achieves concurrent security but adds a common reference string and a reliance on the SGH assumption 3 Furthermore, the Waters signature naturally extends into a hierarchical identity-based signature (cf. 43); applying our construction at level 2 of the resulting signature gives an identity-based blind signature 47. concurrently secure in the common reference string model 4 Alternatively, using the Boyen-Waters group signature [15] at level 1 of the hierarchy and our blind signature at level 2 gives a group blind signature [36] concurrently secure in the common reference string model.

\section{Mathematical Background}

In this paper, we work with bilinear groups, which are cyclic groups $G$ of some finite order that admit a nondegenerate bilinear map $e: G \times G \rightarrow G_{T}$. Because we generalize the concept of a group and work with modules, we are able to describe

\footnotetext{
${ }^{3}$ The efficient range proofs due to Boudot 14 rely on the Strong RSA assumption (due to Baric and Pfitzmann [8]) and require a common reference string. If the scheme of Green and Hohenberger is instantiated with these range proofs then its assumptions and setup model are comparable to those of our scheme, but without providing concurrent security.

${ }^{4}$ One could also obtain an identity-based blind signature through generic composition of our blind signature and an ordinary signature [26].
} 
our scheme without relying on any particular properties of the underlying group (with the caveat, as mentioned above, that the scheme is provably secure only for composite-order groups).

\subsection{Modules}

We first recall the definition of a module; this serves as the foundation for our blind signature scheme, and more specifically for the Groth-Sahai commitments used in our scheme. (See [21, Ch. 10] for further background on modules.)

Definition 2.1. Let $(\mathcal{R},+, \cdot, 0,1)$ be a finite commutative ring. An $\mathcal{R}$-module $A$ is an abelian group $(A,+, 0)$ such that there exists an operator (namely, scalar multiplication) $\mathcal{R} \times A \rightarrow A$, denoted by $(r, x) \mapsto r x$, satisfying the following four properties for all $r, s \in \mathcal{R}$ and $x, y \in A$ :

$-(r+s) x=r x+s x$.

$-r(x+y)=r x+r y$.

$-r(s x)=(r s) x$.

$-1 x=x$.

When $A$ is written multiplicatively our operator becomes exponentiation and the requirements are written as $x^{r+s}=x^{r} \cdot x^{s},(x \cdot y)^{r}=x^{r} \cdot y^{r},\left(x^{r}\right)^{s}=x^{r s}$, and $x^{1}=x$ for all $r, s \in \mathcal{R}$ and $x, y \in A$.

The concept of a module generalizes that of a vector space: when $\mathcal{R}$ is a field, the definitions of an $\mathcal{R}$-module and an $\mathcal{R}$-vector space coincide. The concept of a module also generalizes the concept of an abelian group, as any abelian group can be viewed as a $\mathbb{Z}$-module. If $A$ is isomorphic to $\mathcal{R}^{r}$ as abelian groups, then $r$ is the rank of $A$. When $\mathcal{R}$ is a field, module rank is the same as vector space dimension. In cryptography, we are most used to working with $\mathbb{Z} / n \mathbb{Z}$ - and $\mathbb{F}_{p}$-modules; for example, any finite group of exponent $p$ can be viewed as a $\mathbb{F}_{p}$-module.

\subsection{Groth-Sahai Commitments}

Groth and Sahai [31] devise two types of commitments: commitments to elements in an $\mathcal{R}$-module $A$, and commitments to elements in the ring $\mathcal{R}$. For our purposes, we will need only commitments to bits; we can simplify things even further by always setting $A=G$ for our bilinear group $G$.

To form commitments to module elements, Groth and Sahai define an $\mathcal{R}$ module $B$ and two homomorphisms $\tau: A \rightarrow B$ and $\rho: B \rightarrow A 5$ These maps are defined such that, for some elements $h_{1}, \ldots, h_{m}$ in $B, \rho\left(h_{i}\right)=1$ for all $i$ and $\rho$ is non-trivial for all $x$ that are not contained in $B_{1}:=\left\langle h_{1}, \ldots, h_{m}\right\rangle$. A commitment to $x \in A$ is then defined as $c(x)=\tau(x) \prod_{i=1}^{m} h_{i}^{r_{i}}$ for random values $r_{1}, \ldots, r_{m} \leftarrow$ $\mathcal{R}$. This means that the $h_{i}$ elements act as keys for the commitment scheme, and that the common reference string is $\left(\mathcal{R}, A, B, \tau, \rho, h_{1}, \ldots, h_{m}\right)$. There are two cases:

\footnotetext{
${ }^{5}$ Our notation differs from that of Groth and Sahai, but the ideas are the same.
} 
- Hiding keys: in this case, the $h_{i}$ elements generate the whole module $B$; in other words, $B_{1}=\left\langle h_{1}, \ldots, h_{m}\right\rangle=B$. This implies that $\tau(A) \subseteq B_{1}$, which means that $c(x)$ will be perfectly hiding (as each commitment will be a random element of $B$ ).

- Binding keys: in this case, $B_{1} \neq B$ and $\rho(c)=\rho\left(\tau(x) h^{r}\right)=\rho \circ \tau(x)$ for some restricted space of inputs $x$. To determine what this restricted space is, we see that $c$ will generally reveal the coset of $B_{1}$ where $\tau(x)$ lives. Thus in order for the commitment to be perfectly binding we must restrict the space of inputs $x$ to be the inverse image of $B_{2} \simeq B / B_{1}$; because we know that $B_{1} \neq B$, both $B_{2}$ and $\tau^{-1}\left(B_{2}\right)$ are non-trivial and so this domain restriction is actually meaningful. (Since $B$ is an abelian group, the quotient module is always well-defined.)

It is clear from these definitions that a set of keys cannot be both hiding and binding, as the settings require very different properties of the commitment keys $h_{1}, \ldots, h_{m}$. To get any meaningful blindness properties, however, we need these two settings to be indistinguishable. We therefore require an assumption that implies this indistinguishability; the choice of assumption depends on the instantiation being used.

\section{Security Notions for Blind Signatures}

We define a blind or partially blind signature scheme in the common reference string (CRS) model to be a collection of four protocols: a Setup $\left(1^{k}\right)$ algorithm that outputs the CRS $\sigma_{C R S}$, a $\operatorname{KeyGen}\left(\sigma_{C R S}\right)$ algorithm that outputs the signing key pair $(p k, s k)$, a BlindSign protocol, which consists of an interaction of the form User $\left(\sigma_{C R S}, p k, M\right) \leftrightarrow \operatorname{Signer}\left(\sigma_{C R S}, s k\right.$ ) (in which the signer outputs success if the protocol is successful, and the user outputs success and the unblinded signature $\sigma$ ), and finally a $\operatorname{Verify}\left(\sigma_{C R S}, p k, M, \sigma\right)$ algorithm that outputs accept if the signature is valid and fail if not.

In general, there are two properties that blind and partially blind signatures must satisfy: blindness and one-more unforgeability. Informally, the blindess requirement says that in the process of signing a user's message, the signer does not learn anything abut the message he is signing. The one-more unforgeability requirement says that if the user interacts with the signer $\ell$ times, then he should be able to produce $\ell$ signatures and no more (so in particular, he cannot produce $\ell+1)$. We now describe these properties more formally.

\subsection{Blind Signatures}

Formal definitions of blind signatures were introduced by Juels, Luby, and Ostrovsky [34, although both properties were considered informally in Chaum's original paper on the subject [17, and one-more unforgeability was considered formally in Pointcheval and Stern's work on security arguments for signatures [44. 
In the Juels-Luby-Ostrovsky formalization, the blindness property is defined as follows: the adversary is given a public-private key pair and outputs two messages $M_{0}$ and $M_{1}$. He then engages in two signing protocols with honest users: the first user requests a signature on message $M_{b}$ and the second on message $M_{1-b}$, where $b$ is a random bit unknown to the adversary. The adversary is then given the resulting signatures $\sigma_{0}$ and $\sigma_{1}$, but only if both interactions are successful, and his goal is to guess the bit $b$ (given the messages, the corresponding signatures, and the signing protocol transcripts).

In this paper, we use a stronger version of the blindness property which allows the adversary to generate the signing key pair himself, possibly in a malicious manner. This strengthening was proposed independently in several recent papers 114135.

The one-more unforgeability property can be defined as follows: the adversary is given a public key and engages in multiple executions of the blind signing protocol with a signer; the adversary is able to choose how to interleave the executions. At the end, the adversary is considered successful if he is able to output $\ell+1$ distinct message-signature pairs $\left(M_{1}, \sigma_{1}\right), \ldots,\left(M_{\ell+1}, \sigma_{\ell+1}\right)$, where $\ell$ is the number of executions in which the signer outputs success.

In this definition, two message-signature pairs $\left(M_{i}, \sigma_{i}\right)$ and $\left(M_{j}, \sigma_{j}\right)$ are considered distinct even if $M_{i}=M_{j}$ (so if $\sigma_{i}$ and $\sigma_{j}$ are just two different signatures on the same message) for $i \neq j$. Unfortunately, this means that any signature scheme in which signatures can be re-randomized (like our signature scheme, as we will see in Section (4) will automatically be unable to satisfy one-more unforgeability. We therefore weaken the property by requiring that the adversary be unable to output $\ell+1$ message-signature pairs in which the messages are all distinct 6 This modified definition was also considered recently by Okamoto 41 .

We put all this information together and give a formal definition of security for blind signature schemes in the full version of this paper [39].

\subsection{Partially Blind Signatures}

The security properties of blind signatures can also be extended to partially blind signatures; these formalizations are due to Abe and Okamoto [6]. For partially blind signatures, the adversary outputs two info strings info $o^{(0)}$ and info ${ }^{(1)}$ in addition to its messages $M_{0}$ and $M_{1}$. It then interacts with two separate users in the same manner as before, except this time the first user requests a signature on $M_{b}$ using info $^{(0)}$ and the second requests a signature on $M_{1-b}$ with info info ${ }^{(1)}$. The adversary is given the resulting signatures $\sigma_{0}$ and $\sigma_{1}$ if both interactions were successful and if info ${ }^{(0)}=$ info $^{(1)}$. As before, his goal is to guess the bit $b$.

The one-more unforgeability property is also quite similar to the property for blind signatures; here, the adversary is allowed to choose the info string for each interaction with the signer. The goal is then for the adversary to output an info

\footnotetext{
${ }^{6}$ We observe that blind signatures satisfying this weakened unforgeability property are still sufficient for e-cash and other standard applications of blind signatures.
} 
string info ${ }^{*}$ as well as $\ell+1$ message-signature pairs $\left(M_{1}, \sigma_{1}\right), \ldots,\left(M_{\ell+1}, \sigma_{\ell+1}\right)$, where $\ell$ represents the number of interactions in which the signer output success while using the info string info*.

\section{Underlying Signature Scheme}

As our underlying signature scheme we use a slightly modified version of the Waters signature scheme [4]. Essentially, we just need to generalize the Waters signature scheme by bringing it into the language of modules so that we can use it in combination with Groth-Sahai commitments to create our blind signature scheme.

\subsection{CRS Setup}

For the Waters signature, the required elements for the common reference string are a bilinear group $G$, the target group $G_{T}$ and the bilinear map $e: G \times G \rightarrow G_{T}$, as well as generators $g, u^{\prime}, u_{1}, \ldots, u_{k}$ for $G$, where $k$ denotes the length of the messages to be signed. We now add in the elements discussed in Section 2.2. we start with a ring $\mathcal{R}$ such that $G$ can be interpreted as an $\mathcal{R}$-module. We then add in an $\mathcal{R}$-module $B$, a map $\tau: G \rightarrow B$, a map $\rho: B \rightarrow G$, and a bilinear map $E: B \times B \rightarrow B_{T}$, which also requires us to specify a target module $B_{T}$ and the resulting $\tau_{T}$ and $\rho_{T}$ maps. This means that the CRS will be $\sigma_{\text {sig }}=\left(\mathcal{R}, G, G_{T}, B, B_{T}, e, E, \tau, \tau_{T}, \rho, \rho_{T}, g, u^{\prime}, u_{1}, \ldots, u_{k}\right)$. The relations between all these maps are summarized in the following figure:

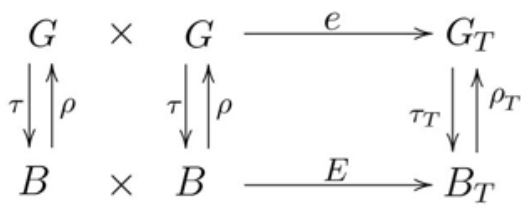

Fig. 1. Commutative diagram for our modules

\subsection{Signing Protocol}

In our generalized Waters signature, the size of the message space will be $\{0,1\}^{k}$ for some value $k$ (for example, to use hash-and-sign with SHA-1 as the hash function we would set $k=160)$. As noted above, the CRS, which is shared between the user and the signer, will contain $k+1$ random generators of $G$.

- Setup $\left(1^{k}\right)$ : Output a tuple $\sigma_{\text {sig }}$ that has been computed as described in the previous section.

- KeyGen $\left(\sigma_{\text {sig }}\right)$ : Pick a random value $\alpha \leftarrow \mathcal{R}$ and set $A=E(\tau(g), \tau(g))^{\alpha}$. The public key will be $p k=A$ and the secret key will be $s k=\alpha$ (actually, $\tau(g)^{\alpha}$ will suffice). 
- $\operatorname{Sign}\left(\sigma_{s i g}, s k, M\right):$ Write the message out bitwise as $M=b_{1} \ldots b_{k}$, and write $s k=\tau(g)^{\alpha}$. Pick a random $r \leftarrow \mathcal{R}$ and compute

$$
S_{1}=\tau(g)^{\alpha}\left(\tau\left(u^{\prime}\right) \prod_{i=1}^{k} \tau\left(u_{i}\right)^{b_{i}}\right)^{r} \quad \text { and } \quad S_{2}=\tau(g)^{-r} .
$$

Output the signature $\sigma=\left(S_{1}, S_{2}\right)$.

- Verify $\left(\sigma_{s i g}, p k, M, \sigma\right)$ : Again, write the message out bitwise as $M=b_{1} \ldots b_{k}$; also write the signature as $\sigma=\left(S_{1}, S_{2}\right)$ and the public key as $p k=A$. Check that these values satisfy the following equation:

$$
E\left(S_{1}, \tau(g)\right) \cdot E\left(S_{2}, \tau\left(u^{\prime}\right) \prod_{i=1}^{k} \tau\left(u_{i}\right)^{b_{i}}\right)=A .
$$

If they do, output accept; else, output fail.

One nice property of the Waters signature (and our extended Waters signature) is that anyone can re-randomize a signature by choosing $s \leftarrow \mathcal{R}$ and computing $S_{1}^{\prime}=S_{1} \cdot\left(\tau\left(u^{\prime}\right) \prod_{i} \tau\left(u_{i}\right)^{b_{i}}\right)^{s}$ and $S_{2}^{\prime}=S_{2} \cdot \tau(g)^{-s}$. Since this results in $S_{1}^{\prime}=\tau(g)^{\alpha}\left(\tau\left(u^{\prime}\right) \prod_{i} \tau\left(u_{i}\right)^{b_{i}}\right)^{r+s}$ and $S_{2}^{\prime}=\tau(g)^{-(r+s)}$, the re-randomization process really does give us a valid signature. In particular, the randomness in the resulting signature $\left(S_{1}^{\prime}, S_{2}^{\prime}\right)$ will be information-theoretically independent from the randomness $r$ chosen by the signer in the signature $\left(S_{1}, S_{2}\right)$.

We recall the computational Diffie-Hellman (CDH) assumption used for the Waters signature:

Assumption 4.1. Let $\mathcal{G}$ be an algorithm that outputs a tuple $(q, G, g)$, where $G$ is a group of order $q$ (not necessarily prime) and $g$ is a generator of $g$. We say that $G$ satisfies the computational Diffie-Hellman assumption if it is computationally infeasible to compute the value $g^{a b}$ given the tuple $\left(g, g^{a}, g^{b}\right)$. More formally, for all PPT adversaries $\mathcal{A}$ there exists a negligible function $\nu(\cdot)$ and a security parameter $k_{0}$ such that the following holds for all $k>k_{0}$ :

$$
\operatorname{Pr}\left[(q, G, g) \leftarrow \mathcal{G}\left(1^{k}\right) ; a, b \leftarrow \mathbb{Z}_{q}: \mathcal{A}\left(g, g^{a}, g^{b}\right)=g^{a b}\right]=\nu(k) .
$$

The Waters signature scheme is existentially unforgeable if $G$ satisfies the CDH assumption. In our extended version, the signature scheme will be existentially unforgeable if $B$ satisfies the $\mathrm{CDH}$ assumption. As the proof is a trivial extension of Waters' proof, we will not include it here.

\section{Our Blind Signature Scheme}

In this section we describe our blind signature scheme. Although we describe only the partially blind setting, our description also encapsulates the fully blind setting, which corresponds to the case $k_{0}=0$. 


\subsection{CRS Setup}

In our CRS we must include all the necessary elements for Groth-Sahai commitments as well as values in the tuple $\sigma_{s i g}$ of Section 4.1. This means our CRS will be $\sigma_{C R S}=\left(\sigma_{\text {sig }}, h_{1}, \ldots, h_{m}\right)$, where the $h_{i}$ elements are binding keys for GrothSahai commitments. Specifically, the elements $h_{i}$ generate a proper submodule $B_{1}$ of the module $B$ used in the Waters signature scheme.

\subsection{The Partially Blind Protocol}

In the following protocol, the user and signer both have access to an info string info. At the end of the protocol, the user obtains a signature on info $\| M$ for a message $M$, while the signer learns nothing beyond the fact that the message $M$ followed the guidelines laid out in info. In addition, the user and the signer will have agreed upon the length of the info string; call it $k_{0}$ for $0 \leq k_{0} \leq k$. Setting $k_{0}=0$ corresponds to a fully blind signature, while setting $k_{0}=k$ corresponds to an ordinary run of the (generalized) Waters signature scheme.

- Setup $\left(1^{k}\right)$ : Output $\sigma_{C R S}$ as described in the previous section (Section 5.1).

- KeyGen $\left(\sigma_{C R S}\right)$ : Same as KeyGen from Section 4.2.

- User $\left(\sigma_{C R S}, p k\right.$, info, $\left.M\right)$ : First write the info string out bitwise, as info = $b_{1} \ldots b_{k_{0}}$, and similarly write the message as $M=b_{k_{0}+1} \ldots b_{k}$. Now, for each $i$ such that $k_{0}<i \leq k$, pick random values $t_{i 1}, \ldots, t_{i m} \leftarrow \mathcal{R}$ and compute

$$
c_{i}=\tau\left(u_{i}\right)^{b_{i}} \cdot \prod_{j=1}^{m} h_{j}^{t_{i j}} \quad \text { and } \quad \pi_{i j}=\left(\tau\left(u_{i}\right)^{2 b_{i}-1} \cdot \prod_{j=1}^{m} h_{j}^{t_{i j}}\right)^{t_{i j}},
$$

where $c_{i}$ acts as a GS commitment to the bit $b_{i}$ and $\vec{\pi}_{i}=\left\{\pi_{i j}\right\}_{j=1}^{m}$ acts as a proof that the value contained in $c_{i}$ is in fact a 0 or a 1 . Send the tuple req $=\left(c_{k_{0}+1}, \vec{\pi}_{k_{0}+1}, \ldots, c_{k}, \vec{\pi}_{k}\right)$ as a request to the issuer (and save some state information state).

- Signer $\left(\sigma_{C R S}, s k\right.$, info, req): First, write info $=b_{1} \ldots b_{k_{0}}$ and $s k=\tau(g)^{\alpha}$. Upon receiving the request, check that each $c_{i}$ is indeed a commitment to a 0 or 1 by checking that

$$
E\left(c_{i}, \tau\left(u_{i}\right)^{-1} c_{i}\right)=\prod_{j=1}^{m} E\left(h_{j}, \pi_{i j}\right)
$$

for each $k_{0}<i \leq k$. If this equation fails to hold for any value of $i$, abort the protocol and output $\perp$. Otherwise, compute the value

$$
c=\tau\left(u^{\prime}\right)\left(\prod_{i=1}^{k_{0}} \tau\left(u_{i}\right)^{b_{i}}\right)\left(\prod_{i=k_{0}+1}^{k} c_{i}\right) .
$$

Finally, pick a random value $r \leftarrow \mathcal{R}$ and compute

$$
K_{1}=\tau(g)^{\alpha} \cdot c^{r}, \quad K_{2}=\tau(g)^{-r}, \quad \text { and } \quad K_{3 j}=h_{j}^{-r} \quad \text { for } 1 \leq j \leq m .
$$

Set $\vec{K}_{3}=\left\{K_{3 j}\right\}_{j=1}^{m}$, send the tuple $\left(K_{1}, K_{2}, \vec{K}_{3}\right)$ back to the user, and output success and info. 
- User $\left(\right.$ state, $\left.\left(K_{1}, K_{2}, \vec{K}_{3}\right)\right)$ : First, check that $K_{2}$ and $\vec{K}_{3}$ were formed properly by checking satisfiability of

$$
E\left(K_{3 j}, \tau(g)\right)=E\left(K_{2}, h_{j}\right)
$$

for each $1 \leq j \leq m$. If this equation does not verify for some $j$, abort and output $\perp$. Otherwise, unblind the signature by computing

$$
S_{1}=K_{1} \prod_{i=k_{0}+1}^{k} \prod_{j=1}^{m} K_{3 j}^{t_{i j}} \quad \text { and } \quad S_{2}=K_{2} .
$$

Next verify that this is a valid signature on info $\| M$ by running Verify $\left(\sigma_{C R S}\right.$, $p k$, info $\left.\| M,\left(S_{1}, S_{2}\right)\right)$. If this step outputs fail, abort the protocol and output $\perp$. If it outputs accept, however, re-randomize the signature by choosing a random value $s \leftarrow \mathcal{R}$ and computing

$$
S_{1}^{\prime}=S_{1}\left(\tau\left(u^{\prime}\right) \prod_{i=1}^{k} \tau\left(u_{i}\right)^{b_{i}}\right)^{s} \quad \text { and } \quad S_{2}^{\prime}=S_{2} \cdot \tau(g)^{-s} .
$$

The final signature is $\sigma=\left(S_{1}^{\prime}, S_{2}^{\prime}\right)$; output $\sigma$ as well as info and success. - Verify $\left(\sigma_{C R S}, p k, M, \sigma\right)$ : Same as Verify from Section 4.2

Theorem 5.1. The blind signature scheme outlined above is correct and partially blind, under the assumption that the $h_{i}$ values in the hiding and binding settings are indistinguishable.

The proof of Theorem 5.1 appears in the full version of this paper 39. The theorem demonstrates correctness and (partial) blindness, but it does not show one-more unforgeability. In order to prove this last property, we need to define two properties of pairings, adapted from Freeman [24, §3] for our purposes:

Definition 5.2. A pairing $E: B \times B \rightarrow B_{T}$ is cancelling if there exists a decomposition $B=B_{1} \times B_{2}$ such that $E\left(b_{1}, b_{2}\right)=1$ for all $b_{1} \in B_{1}, b_{2} \in B_{2}$.

Definition 5.3. A pairing $E: B \times B \rightarrow B_{T}$ is projecting if there exists a decomposition $B=B_{1} \times B_{2}$, a submodule $B_{T}^{\prime} \subset B_{T}$, and homomorphisms $\pi: B \rightarrow B$ and $\pi_{T}: B_{T} \rightarrow B_{T}$, such that $B_{1} \subseteq \operatorname{ker}(\pi), \pi(x)=x$ for $x \in B_{2}, B_{T}^{\prime} \subseteq \operatorname{ker}\left(\pi_{T}\right)$, and $\pi_{T}(E(x, y))=E(\pi(x), \pi(y))$ for all $x, y \in B$.

As we will see below, the pairing $E$ has both of these properties (with respect to the same decomposition $B=B_{1} \times B_{2}$ ) when instantiated using compositeorder groups under the Subgroup Hiding (SGH) assumption. Because SGH also provides the necessary indistinguishability properties, we obtain the following theorem, a proof of which can be found in the full version of this paper [39]:

Theorem 5.4. The blind signature scheme outlined above is one-more unforgeable under the SGH assumption and the assumption that the modified Waters signature scheme in Section 4 is existentially unforgeable on the submodule $B_{2} \subseteq B$. 


\subsection{Instantiation under the SGH Assumption}

We first recall the Subgroup Hiding (SGH) assumption:

Assumption 5.5 ([12]). Let $\mathcal{G}$ be an algorithm that outputs a tuple $(p, q, G$, $\left.G_{T}, e\right)$ such that $G$ and $G_{T}$ are both groups of order $n=p q$ and $e: G \times G \rightarrow G_{T}$ is a nondegenerate bilinear map. We say that $G$ satisfies the Subgroup Hiding assumption if it is computationally infeasible to distinguish between an element of $G$ and an element of $G_{q}$. More formally, for all PPT adversaries $\mathcal{A}$ there exists a negligible function $\nu(\cdot)$ and a security parameter $k_{0}$ such that the following holds for all $k>k_{0}$ :

$$
\begin{aligned}
& \mid \operatorname{Pr}\left[\left(p, q, G, G_{T}, e\right) \leftarrow \mathcal{G}\left(1^{k}\right) ; n=p q ; x \leftarrow G: \mathcal{A}\left(n, G, G_{T}, e, x\right)=0\right] \\
& -\operatorname{Pr}\left[\left(p, q, G, G_{T}, e\right) \leftarrow \mathcal{G}\left(1^{k}\right) ; n=p q ; x \leftarrow G: \mathcal{A}\left(n, G, G_{T}, e, x^{p}\right)=0\right] \mid<\nu(k) .
\end{aligned}
$$

To instantiate our blind signature scheme under this assumption, we use a group $G$ of order $n=p q$ with $p, q$ prime. We define $B=G$ and $\tau$ to be the identity map; this means that we can use $E=e$. We need only one $h_{i}$ element, namely an $h_{1}$ such that $h_{1}$ generates $G_{q}$ in the binding setting and $h_{1}$ generates the whole group $G$ in the hiding setting. The SGH assumption tells us that these choices of $h_{1}$ are indistinguishable. We can also describe our $\rho$ map as $\rho\left(c_{i}\right)=c_{i}^{q}=\left(u_{i}^{q}\right)^{b_{i}}$ since $h_{1}$ has order $q$. Because the $u_{i}$ are all generators for $G$ and therefore $u_{i}^{q} \neq 1$, we see that the $\rho$ map will indeed reveal the bit $b_{i}$.

Because $h_{1}$ will generate either $G$ or $G_{q}$, we have $B=G_{p} \times G_{q}$, which means (looking back at the statement of Theorem 5.4) that we assume for the security of our blind signature that $\mathrm{CDH}$ is hard in $G_{p}$. To see that the pairing $e$ is cancelling, note that every element of $G_{p}$ can be written as $a=g^{\alpha q}$ for some $\alpha \in \mathbb{F}_{p}$ and every element of $G_{q}$ can be written as as $b=g^{\beta p}$ for some $\beta \in \mathbb{F}_{q}$. Then $e(a, b)=e\left(g^{\alpha q}, g^{\beta p}\right)=e\left(g^{\alpha \beta p q}, g\right)=e\left(\left(g^{n}\right)^{\alpha \beta}, g\right)=1$ because $G$ has order $n$. To see that $e$ is projecting, note that there exists a $\lambda \in \mathbb{Z}_{n}$ such that $\lambda \equiv 1 \bmod p$ and $\lambda \equiv 0 \bmod q$, and that furthermore this value is efficiently computable (given the factorization of $n$ ) using the Chinese Remainder Theorem. Thus exponentiating by $\lambda$ cancels out the $G_{q}$ component of a group element while leaving the $G_{p}$ component unchanged. This allows us to define $\pi(z)=z^{\lambda}$ for $z \in G$ and $\pi_{T}\left(z_{T}\right)=\left(z_{T}\right)^{\lambda}$ for $z_{T} \in G_{T}$. These maps are easily seen to satisfy the projecting properties.

Finally, to compute the value $h_{1}$ we can set $h_{1}=g$ in the hiding setting and $h_{1}=g^{p}$ in the binding setting. This means that, as with the map $\rho$, the factorization of $n$ will be required as a trapdoor to compute $h_{1}$.

The obvious downside of using our scheme under the SGH assumption is the use of a composite-order group, which necessitates a common reference string generated by a trusted third party 7 The upside, on the other hand, is that the

\footnotetext{
${ }^{7}$ It is an open problem to replace the trusted third party with an efficient secure multiparty computation protocol for computing the CRS.
} 
scheme is as efficient as possible under this assumption, as each part of the signature involves only one group element 8

\section{Converting to a Prime-Order Setting}

In this section we argue that our scheme cannot be instantiated securely under a natural usage of the $k$-Linear family of assumptions in prime-order groups. The $k$-Linear family generalizes the Decision Diffie-Hellman and Decision Linear [1] assumptions (which can be recovered by setting $k=1$ or 2 , respectively) and is defined as follows:

Assumption 6.1 (45,33]). Let $\mathcal{G}$ be a generation algorithm that outputs a tuple $(p, G, g)$ such that $p$ is prime, $G$ is a group of order $p$, and $g$ is a generator of $G$. We say that $G$ satisfies the $k$-Linear assumption if it is computationally infeasible to distinguish between tuples of the form $\left(g^{\alpha_{1}}, \ldots, g^{\alpha_{k+1}}, g^{\alpha_{1} r_{1}}, \ldots, g^{\alpha_{k} r_{k}}\right.$, $\left.g^{\alpha_{k+1} \sum_{i=1}^{k} r_{i}}\right)$ and tuples of the form $\left(g^{\alpha_{1}}, \ldots, g^{\alpha_{k+1}}, g^{\alpha_{1} r_{1}}, \ldots, g^{\alpha_{k+1} r_{k+1}}\right)$ for random $\alpha_{i}, r_{i} \leftarrow \mathbb{F}_{p}$. More formally, for all PPT adversaries $\mathcal{A}$ there exists a negligible function $\nu(\cdot)$ and a security parameter $k_{0}$ such that the following holds for all $k>k_{0}$ :

$$
\begin{aligned}
\mid \operatorname{Pr}\left[\begin{array}{c}
(p, G, g) \leftarrow \mathcal{G}\left(1^{k}\right) \\
\alpha_{1}, \ldots, \alpha_{k+1} \stackrel{R}{\leftarrow} \mathbb{F}_{p}: \mathcal{A}\left(g^{\alpha_{1}}, \ldots, g^{\alpha_{k+1}}, g^{\alpha_{1} r_{1}}, \ldots, g^{\alpha_{k+1} \sum_{i=1}^{k} r_{i}}\right)=0 \\
r_{1}, \ldots, r_{k} \stackrel{R}{\leftarrow} \mathbb{F}_{p}
\end{array}\right] \\
-\operatorname{Pr}\left[\begin{array}{l}
(p, G, g) \leftarrow \mathcal{G}\left(1^{k}\right) \\
\alpha_{1}, \ldots, \alpha_{k+1} \stackrel{R}{\leftarrow} \mathbb{F}_{p}: \mathcal{A}\left(g^{\alpha_{1}}, \ldots, g^{\alpha_{k+1}}, g^{\alpha_{1} r_{1}}, \ldots, g^{\alpha_{k+1} r_{k+1}}\right)=0 \\
r_{1}, \ldots, r_{k+1} \stackrel{\mathrm{R}}{\leftarrow} \mathbb{F}_{p}
\end{array}\right]<\nu(k),
\end{aligned}
$$

Let $G$ be a bilinear group of prime order $p$ with a pairing $e: G \times G \rightarrow G_{T}$. When we refer to a "natural" use of the $k$-Linear assumption, we mean that we define the module $B$ to be $G^{k+1}$ and the module $B_{1}$ to be generated by $k$ elements of $B$ that span a rank- $k$ submodule. Indeed, one way to interpret the $k$-Linear assumption is that a random element in the submodule $B_{1}$ of $G^{k+1}$ generated by elements of the form $\left(1, \ldots, 1, g^{\alpha_{i}}, 1, \ldots, 1, g\right)$ for $i=1, \ldots, k$ is indistinguishable from a random element of $G^{k+1}$. Our use of the assumption generalizes this interpretation only slightly, by randomizing the generators of $B_{1}$. Note that in our setup the quotient module $B_{2}=B / B_{1}$ has $\mathbb{F}_{p}$-rank 1 .

Following Freeman [24, §2], we define a (symmetric) pairing on $B$ by setting $B_{T}=\left(G_{T}\right)^{m}$ for some integer $m$ and choosing $(k+1) \times(k+1)$ (symmetric) matrices $E^{(\ell)}$ over $\mathbb{F}_{p}$ for $\ell=1, \ldots, m$. We then set the $\ell$ th component of the pairing to be

$$
E\left(\left(g_{1}, \ldots, g_{k+1}\right),\left(h_{1}, \ldots, h_{k+1}\right)\right)^{(\ell)}:=\prod_{i, j=1}^{k+1} e\left(g_{i}, h_{j}\right)^{e_{i j}^{(\ell)}},
$$

\footnotetext{
${ }^{8}$ Of course, the number of bits taken to represent the composite-order group element, approximately 1024, is much larger than it would be for a prime-order group element, which can be as small as 160 bits (at the 80-bit security level).
} 
where $e_{i j}^{(\ell)}$ denotes the $(i, j)$-th entry of $E^{(\ell)}$. The connection between this setup and the $k$-Linear assumption is given by the following theorem:

Theorem 6.2 ([24, Theorem 2.5]). Let $G, B, B_{1}, B_{T}$ be as described above, with $B_{1}$ a uniformly random rank- $k$ submodule of $B$. If $G$ satisfies the $k$-Linear assumption, then a random element of $B_{1}$ is computationally indistinguishable from a random element in $B$.

While any scheme based on Groth-Sahai proofs requires the projecting property of Definition 5.3 and the indistinguishability of elements in $B_{1}$ and $B$ (i.e., the indistinguishability of hiding and binding commitment keys), our scheme also requires the cancelling property of Definition 5.2. In the remainder of this section, we show that for any $k$, the three properties (projecting, cancelling, and key indistinguishability) cannot simultaneously be obtained in prime-order groups using the $k$-Linear assumption as described above, except with negligible probability (over the choice of the module $B_{1}$ ).

We start by showing that the image of a symmetric pairing on a group $G$ of prime order $p$ must also have order $p$. In what follows, we denote by $E(B, B)$ the submodule of $B_{T}$ generated by all elements of the form $E(x, y)$ for $x, y \in B$.

Lemma 6.3. If $G$ is a group of prime order $p$ and $e: G \times G \rightarrow G_{T}$ is a nondegenerate symmetric bilinear map, then the order of $e(G, G)$ is $p$.

Proof. We first observe that $e(G, G)$ has exponent $p$; to see this, note that since $G$ has order $p$, we have $e(x, y)^{p}=e\left(x^{p}, y\right)=e(1, y)=1$ for any $x, y \in G$. Since $e(G, G)$ has exponent $p$, any element is of the form $z=\prod_{i} e\left(x_{i}, y_{i}\right)^{c_{i}}$ for $x_{i}, y_{i} \in G$ and $c_{i} \in \mathbb{F}_{p}$. Since $G$ is cyclic, we can write $x_{i}=g^{a_{i}}$ and $y_{i}=$ $g^{b_{i}}$ for a generator $g$ and unique $a_{i}, b_{i} \in \mathbb{F}_{p}$. By bilinearity, we can write $z=$ $e(g, g)^{\sum_{i} a_{i} b_{i} c_{i}}$, and therefore $e(G, G)$ is a cyclic group generated by $e(g, g)$; the nondegeneracy of $e$ implies that $e(g, g)$ has order $p$.

Lemma 6.3 shows that by replacing $G_{T}$ with $e(G, G)$, we may assume without loss of generality that $G_{T}$ has order $p$. We make this assumption in the remainder of the section. We will also assume that the values used to define the pairing $E$ on the module $B$ are independent of the submodules $B_{1}$ and $B_{2}$; if they are not independent, then the fact that they are related to the (publicly known) generators of $B_{1}$ gives an adversary information about $B_{1}$ that could be used to break the indistinguishability assumption. Similarly, if the pairing depends on $B_{2}$, then the adversary may be able to use this information to compute an element $y \in B_{2}$; then given an element $x$ in either $B_{1}$ or $B$, he could compute $E(x, y)$ and conclude that $x \in B_{1}$ if and only if the resulting value is 1 .

We can now show that in the prime-order setting our indistinguishability restrictions on $B$ and its submodules will, with high probability, yield a pairing $E$ that can be either projecting or cancelling, but not both at the same time. Our approach is to construct a cancelling pairing and then show that it implies that the image of the pairing $E$ is of order $p$. We will then show that this implies that the pairing cannot satisfy the projecting property. 
In general, there are two methods in the literature for cancelling paired group elements. As seen in Section 5.3 the cancelling in the composite setting is fairly straightforward: it follows from the fact that the orders of the $G_{p}$ and $G_{q}$ subgroups are relatively prime. In a prime-order setting this is not an option, as every component (i.e., $G, G_{T}, B, B_{1}, B_{2}, B_{T}$ ) has exponent $p$. We therefore need to use linear combinations of exponents in order to successfully cancel elements. Our next result can be interpreted as showing that forming these linear combinations requires us to combine elements in the pairing and thus shrink the size of the pairing's image. To simplify notation, we state the proposition relative to the $(k-1)$-Linear assumption.

Proposition 6.4. Let $G$ be a bilinear group of prime order $p$ with pairing e: $G \times$ $G \rightarrow G_{T}$. Let $B$ be the rank-k $G$-module $G^{k}$, let $B_{T}=\left(G_{T}\right)^{m}$ for some positive integer $m$, and let $E: B \times B \rightarrow B_{T}$ be a nondegenerate pairing defined as in (5). If $B_{1}$ is a uniformly random rank-(k-1) submodule of $B$ and $E$ is a cancelling pairing that is independent of the decomposition $B=B_{1} \times B_{2}$, then $e(B, B)$ has order $p$ with overwhelming probability.

Proof. To start, we write elements in $B$ as either $\mathbf{a}=\left(a_{1}, \ldots, a_{k}\right)$ or $\mathbf{b}=$ $\left(b_{1}, \ldots, b_{k}\right)$ with $a_{i}, b_{i} \in G$. Equivalently, we can fix a generator $g$ of $G$ and write $\mathbf{a}=\left(g^{\alpha_{1}}, \ldots, g^{\alpha_{k}}\right)$ and $\mathbf{b}=\left(g^{\beta_{1}}, \ldots, g^{\beta_{k}}\right)$ for exponents $\alpha_{i}, \beta_{i} \in \mathbb{F}_{p}$. As we saw in (5) above, the element $E(\mathbf{a}, \mathbf{b}) \in B_{T}$ is a tuple of elements of $G_{T}$, in which each entry is of the form $T=\prod_{i, j} e\left(a_{i}, b_{j}\right)^{e_{i j}}$. By assumption, the coefficients $e_{i j} \in \mathbb{F}_{p}$ are independent of the $\alpha_{i}$ and $\beta_{i}$ values.

Suppose that $\mathbf{a} \in B_{1}$ and $\mathbf{b} \in B_{2}$; let us see what we require in order to have $T=1$. Let $\mathbf{a}_{1}, \ldots, \mathbf{a}_{k-1}$ be a set of generators of $B_{1}$, and write $\mathbf{a}_{u}=$ $\left(g^{\alpha_{u 1}}, \ldots, g^{\alpha_{u, k-1}}\right)$ for $u=1, \ldots, k-1$. Then a general element of $B_{1}$ is given by $\mathbf{a}=\mathbf{a}^{r_{1}} \cdots \mathbf{a}^{r_{k-1}}$ for arbitrary $r_{1}, \ldots, r_{k-1} \in \mathbb{F}_{p}$. Since $B_{1}$ has rank $k-1$, the submodule $B_{2}$ has rank 1 and a general element of $B_{2}$ is given by $\mathbf{b}=$ $\left(g^{\beta_{1} t}, \ldots, g^{\beta_{k} t}\right)$ for some fixed $\beta_{1}, \ldots, \beta_{k} \in \mathbb{F}_{p}$ and arbitrary $t \in \mathbb{F}_{p}$. Looking back at how our element $T$ is computed in (5), we can see that the condition $T=1$ is equivalent to

$$
\sum_{u} r_{u}\left(\sum_{i} \alpha_{u i}\left(\sum_{j} e_{i j} \beta_{j} t\right)\right)=0
$$

In matrix notation, this is $\vec{r} \cdot A \cdot E \cdot \vec{b} \cdot t=0$, where $\vec{r}$ is the row vector $\left(r_{1}, \ldots, r_{k-1}\right)$, $E=\left[e_{i j}\right]$ is the $k \times k$ matrix specifying the pairing coefficients (denoted $E^{(\ell)}$ in (5)), $A=\left[\alpha_{u i}\right]$ is the $(k-1) \times k$ matrix whose rows are the vectors corresponding to the generators of $B_{1}$, and $\vec{b}$ is the column vector $\left(\beta_{1}, \ldots, \beta_{k}\right)$. Because this requirement must hold for all values of $\vec{r}$ and $t$, we can further reduce the equation to $A \cdot E \cdot \vec{b}=0$. We now consider two different cases: when $E$ is invertible and when $E$ is singular.

We first consider the case where $E$ is singular. The cancelling property requires that $A \cdot E \cdot \vec{b}=0$. If $E \cdot \vec{b}=0$, then the pairing is degenerate in this component, as any element paired with $\vec{b}$ will be 1 . Therefore, this cannot be the only type 
of element in the pairing tuple, or else the entire pairing would be degenerate. On the other hand, if $E \cdot \vec{b} \neq 0$, then since $A \cdot E \cdot \vec{b}=0$, we see that $E \cdot \vec{b}$ is a nonzero vector in both the image of $E$ and the kernel of $A$.

Next we consider the case where $E$ is invertible, and consider not only the element $T$ but also another element $T^{\prime}$ in the target tuple. The element $T^{\prime}$ will have its own associated coefficient matrix $E^{\prime}$, with the requirement that $A \cdot E^{\prime} \cdot \vec{b}=0$. Then we have $A \cdot E \cdot \vec{b}=A \cdot E^{\prime} \cdot \vec{b}=0$, which implies that $\vec{b}$ is contained in both the kernel of $A \cdot E$ and the kernel of $A \cdot E^{\prime}$. Since $A$ has rank $k-1$ and we are assuming $E$ to be invertible, we know that the dimension of $\operatorname{ker}(A \cdot E)$ is 1 . Furthermore, since $E$ is invertible we can write

$$
A \cdot E^{\prime} \cdot \vec{b}=A \cdot\left(E \cdot E^{-1}\right) \cdot E^{\prime} \cdot \vec{b}=A \cdot E \cdot\left(E^{-1} \cdot E^{\prime} \cdot \vec{b}\right)=0
$$

which implies that $E^{-1} E^{\prime} \cdot \vec{b}$ is also contained in the kernel of $A \cdot E$. Since this kernel is one-dimensional, $E^{-1} E^{\prime} \cdot \vec{b}$ must be a constant multiple of $\vec{b}$; i.e., $E^{-1} E^{\prime} \cdot \vec{b}=\lambda \cdot \vec{b}$ for some $\lambda \in \mathbb{F}_{p}$ and $\vec{b}$ is an eigenvector of $E^{-1} E^{\prime}$.

We now observe that because $A$ has rank $k-1$, its kernel has rank one; furthermore, choosing a rank- $(k-1)$ submodule $B_{1}$ is equivalent to choosing the one-dimensional subspace $\operatorname{ker}(A)$. Since $E$ is invertible and independent of $B_{1}$, this is equivalent to choosing the one-dimensional subspace $\operatorname{ker}(A \cdot E)$. Let $\vec{u}$ be any vector in $\operatorname{ker}(A \cdot E)$. Then $\vec{u}=\gamma \cdot \vec{b}$ for some $\gamma \in \mathbb{F}_{p}$, and our analysis above shows that $\vec{u}$ is an eigenvector of $E^{-1} E$. Since $\operatorname{ker}(A \cdot E)$ can contain any nonzero vector $\vec{u}$, this implies that every vector is an eigenvector of $E^{-1} E$. Therefore $E^{-1} E^{\prime}$ must be a diagonal matrix with the same value in each diagonal entry; in other words, we have $E^{-1} E^{\prime}=c I$ for some constant $c \in \mathbb{F}_{p}$. Thus we have $E^{\prime}=c I \cdot E=c \cdot E$, and so $T^{\prime}=T^{c}$.

It remains only to put everything together. Let $E^{(\ell)}$ be the coefficient matrix from (5) used to compute the $\ell$ th component of the pairing. Our argument above shows that if one of the matrices $E^{(\ell)}$ is invertible, then all matrices $E^{\left(\ell^{\prime}\right)}$ are constant multiples of $E^{(\ell)}$, and therefore the order of $e(B, B)$ is the same as the order of $e(G, G)=G_{T}$, which is $p$. Thus if the pairing $E$ is cancelling and the order of $e(B, B)$ is greater than $p$, then none of the matrices $E^{(\ell)}$ can be invertible.

Now suppose all of the $E^{(\ell)}$ are singular. Our consideration of this case above shows that if the pairing $E$ is cancelling, then there must be some matrix $E^{(\ell)}$ with $\operatorname{ker}(A) \cap \operatorname{im}\left(E^{(\ell)}\right) \neq\{0\}$. As noted above, choosing the module $B_{1}$ is equivalent to choosing the one-dimensional subspace $\operatorname{ker} A$. Since $E^{(\ell)}$ is not invertible, we have $\operatorname{dim}\left(\operatorname{im}\left(E^{(\ell)}\right)\right) \leq k-1$. Thus the number of one-dimensional subspaces in $\operatorname{im}\left(E^{(\ell)}\right)$ is at most $\left(p^{k-1}-1\right) /(p-1)$, while the number of one-dimensional subspaces in $\mathbb{F}_{p}^{k}$ is $\left(p^{k}-1\right) /(p-1)$. We conclude that the probability (taken over a uniformly random choice of $\operatorname{ker}(A)$ and thus also of $A$ ) that $\operatorname{ker}(A)$ has nontrivial intersection with the image of $E^{(\ell)}$ is at most $\left(p^{k-1}-1\right) /\left(p^{k}-1\right)<2 / p$. Taking a union bound, we conclude that the probability that $\operatorname{ker}(A) \cap \operatorname{im}\left(E^{(\ell)}\right) \neq 0$ for some $\ell$ is at most $2 m / p$, which is negligible.

Putting all this together, we can prove our main theorem: 
Theorem 6.5. Let $G$ be a bilinear group of prime order $p$ with pairing $e: G \times$ $G \rightarrow G_{T}$. Let $B$ be the rank- $k G$-module $G^{k}$, let $B_{T}=\left(G_{T}\right)^{m}$ for some positive integer $m$, and let $E: B \times B \rightarrow B_{T}$ be a nondegenerate pairing defined as in (5). If $B_{1}$ is a uniformly random rank- $(k-1)$ submodule of $B$ and $E$ is a cancelling pairing that is independent of the decomposition $B=B_{1} \times B_{2}$, then with overwhelming probability the pairing $E$ cannot be projecting (with respect to the same decomposition $\left.B=B_{1} \times B_{2}\right)$.

Proof. By Proposition 6.4, we know that if $E$ is cancelling, then $E(B, B)$ has order $p$ with overwhelming probability. This means that $E(B, B)$ is cyclic and any nonzero element is a generator.

Suppose $E$ is projecting and choose some $x \in B_{1}$. Since $E$ is nondegenerate, there is some $y \in B$ such that $E(x, y) \neq 1$. Now the projecting property implies that $\pi_{T}(E(x, y))=E(\pi(x), \pi(y))=E(1, \pi(y))=1$. Since $E(x, y)$ generates $E(B, B)$, we conclude that $\pi_{T}(E(B, B))=\{1\}$.

On the other hand, now choose some $x^{\prime} \in B_{2}$. Then there is some $y^{\prime} \in$ $B$ such that $E\left(x^{\prime}, y^{\prime}\right) \neq 1$. Furthermore, the cancelling property implies that without loss of generality we can assume $y^{\prime} \in B_{2}$. The projecting property now implies that $\pi_{T}\left(E\left(x^{\prime}, y^{\prime}\right)\right)=E\left(\pi\left(x^{\prime}\right), \pi\left(y^{\prime}\right)\right)=E\left(x^{\prime}, y^{\prime}\right) \neq 1$, so we conclude that $\pi_{T}(E(B, B))=E(B, B)$, contradicting our conclusion above.

\section{Conclusions and Open Problems}

In this paper we have shown that there are limitations on transformations of pairing-based cryptosystems from composite- to prime-order groups. In particular, we have given evidence that two properties of composite-order pairings identified by Freeman — cancelling and projecting — cannot be simultaneously obtained in prime-order groups.

Specifically, we have shown that a pairing defined in a natural way with subgroup hiding provided by the Decision Linear assumption can be both cancelling and projecting with only negligible probability. As evidence that both properties are sometimes called for simultaneously, we have presented a natural cryptographic scheme whose proof of security calls for a pairing that is both cancelling and projecting. This scheme is a practical round-optimal blind (and partially blind) signature secure in the common reference string model, under mild assumptions and without random oracles.

Many open questions remain. First, we would like either to generalize our result so it applies to a wider class of pairings constructed from prime order groups (possibly including asymmetric pairings), or instead to show that no such generalization is possible by exhibiting a pairing in prime-order groups that is simultaneously projecting and cancelling. Second, we have given evidence that our specific proof strategy for our blind signature scheme is unlikely to generalize to prime-order groups, but have not settled the question of whether our scheme when instantiated in prime-order groups is in fact provably secure (by means of a different, ad-hoc proof) or insecure (i.e., actually susceptible to attack). Finally, it is interesting to consider whether a more general procedure (not relying on 
Freeman's properties) can be used to transform every composite-order scheme to a prime-order one, or whether some schemes provably cannot be so transformed.

Acknowledgements. We are grateful to Melissa Chase for helpful discussions about this work and to our anonymous reviewers for their helpful comments. The first author is supported in part by a MURI grant administered by the Air Force Office of Scientific Research and in part by a graduate fellowship from the Charles Lee Powell Foundation. The third author is supported by an NSF Mathematical Sciences Postdoctoral Research Fellowship.

\section{References}

1. Abdalla, M., Namprempre, C., Neven, G.: On the (im)possibility of blind message authentication codes. In: Pointcheval, D. (ed.) CT-RSA 2006. LNCS, vol. 3860, pp. 262-279. Springer, Heidelberg (2006)

2. Abe, M., Fuchsbauer, G., Groth, J., Haralambiev, K., Ohkubo, M.: Structurepreserving signatures and commitments to group elements. In: Rabin, T. (ed.) CRYPTO 2010. LNCS, vol. 6223, pp. 209-236. Springer, Heidelberg (2010)

3. Abe, M., Fujisaki, E.: How to date blind signatures. In: Kim, K., Matsumoto, T. (eds.) ASIACRYPT 1996. LNCS, vol. 1163, pp. 244-251. Springer, Heidelberg (1996)

4. Abe, M., Haralambiev, K., Ohkubo, M.: Signing on elements in bilinear groups for modular protocol design. Cryptology ePrint Archive, Report 2010/133 (2010), http://eprint.iacr.org/

5. Abe, M., Ohkubo, M.: A framework for universally composable non-committing blind signatures. In: Matsui, M. (ed.) ASIACRYPT 2009. LNCS, vol. 5912, pp. 435-450. Springer, Heidelberg (2009)

6. Abe, M., Okamoto, T.: Provably secure partially blind signatures. In: Bellare, M. (ed.) CRYPTO 2000. LNCS, vol. 1880, pp. 271-286. Springer, Heidelberg (2000)

7. Ballard, L., Green, M., de Medeiros, B., Monrose, F.: Correlation-resistant storage via keyword-searchable encryption. Cryptology ePrint Archive, Report 2005/417, (2005), http://eprint.iacr.org/

8. Baric, N., Pfitzmann, B.: Collision-free accumulators and fail-stop signature schemes without trees. In: Fumy, W. (ed.) EUROCRYPT 1997. LNCS, vol. 1233, pp. 480-494. Springer, Heidelberg (1997)

9. Bellare, M., Namprempre, C., Pointcheval, D., Semanko, M.: The power of RSA inversion oracles and the security of Chaum's RSA-based blind signature scheme. In: Syverson, P.F. (ed.) FC 2001. LNCS, vol. 2339, pp. 319-338. Springer, Heidelberg (2002)

10. Boldyreva, A.: Threshold signature, multisignature and blind signature schemes based on the gap-Diffie-Hellman-group signature scheme. In: Desmedt, Y. (ed.) PKC 2003. LNCS, vol. 2567, pp. 31-46. Springer, Heidelberg (2002)

11. Boneh, D., Boyen, X., Shacham, H.: Short group signatures. In: Franklin, M. (ed.) CRYPTO 2004. LNCS, vol. 3152, pp. 41-55. Springer, Heidelberg (2004)

12. Boneh, D., Goh, E.-J., Nissim, K.: Evaluating 2-DNF formulas on ciphertexts. In: Kilian, J. (ed.) TCC 2005. LNCS, vol. 3378, pp. 325-341. Springer, Heidelberg (2005)

13. Boneh, D., Rubin, K., Silverberg, A.: Finding composite order ordinary elliptic curves using the Cocks-Pinch method. Cryptology ePrint Archive, Report 2009/533, (2009), http://eprint.iacr.org/2009/533 
14. Boudot, F.: Efficient proofs that a committed number lies in an interval. In: Preneel, B. (ed.) EUROCRYPT 2000. LNCS, vol. 1807, pp. 431-444. Springer, Heidelberg (2000)

15. Boyen, X., Waters, B.: Compact group signatures without random oracles. In: Vaudenay, S. (ed.) EUROCRYPT 2006. LNCS, vol. 4004, pp. 427-444. Springer, Heidelberg (2006)

16. Cao, T., Lin, D., Xue, R.: A randomized RSA-based partially blind signature scheme for electronic cash. Computers and Security 24(1), 44-49 (2005)

17. Chaum, D.: Blind signatures for untraceable payments. In: Chaum, D., Rivest, R., Sherman, A. (eds.) Proceedings of Crypto 1982, pp. 199-204. Plenum Press, New York (1983)

18. Chaum, D.: Blind signature system (abstract). In: Chaum, D. (ed.) Proceedings of Crypto 1983, p. 153. Plenum Press, New York (1984)

19. Chaum, D.: Elections with unconditionally-secret ballots and disruption equivalent to breaking RSA. In: Günther, C. (ed.) EUROCRYPT 1988. LNCS, vol. 330, pp. 177-182. Springer, Heidelberg (1988)

20. Chaum, D., Fiat, A., Naor, M.: Untraceable electronic cash. In: Goldwasser, S. (ed.) CRYPTO 1988. LNCS, vol. 403, pp. 319-327. Springer, Heidelberg (1990)

21. Dummit, D., Foote, R.: Abstract Algebra, 2nd edn. Prentice-Hall, Upper Saddle River (1999)

22. Fischlin, M.: Round-optimal composable blind signatures in the common reference string model. In: Dwork, C. (ed.) CRYPTO 2006. LNCS, vol. 4117, pp. 60-77. Springer, Heidelberg (2006)

23. Freeman, D., Scott, M., Teske, E.: A taxonomy of pairing-friendly elliptic curves. J. Cryptology 23(2), 224-280 (2010)

24. Freeman, D.M.: Converting pairing-based cryptosystems from composite-order groups to prime-order groups. In: Gilbert, H. (ed.) EUROCRYPT 2010. LNCS, vol. 6110, pp. 44-61. Springer, Heidelberg (2010)

25. Fuchsbauer, G.: Automorphic signatures in bilinear groups and an application to round-optimal blind signatures. Cryptology ePrint Archive, Report 2009/320 (2009), http://eprint.iacr.org/

26. Galindo, D., Herranz, J., Kiltz, E.: On the generic construction of identity-based signatures with additional properties. In: Lai, X., Chen, K. (eds.) ASIACRYPT 2006. LNCS, vol. 4284, pp. 178-193. Springer, Heidelberg (2006)

27. Garg, S., Sahai, A., Waters, B.: Efficient fully collusion-resilient traitor tracing scheme. Cryptology ePrint Archive, Report 2009/532 (2009), http://eprint.iacr.org/2009/532

28. Green, M., Hohenberger, S.: Blind identity-based encryption and simulatable oblivious transfer. In: Kurosawa, K. (ed.) ASIACRYPT 2007. LNCS, vol. 4833, pp. 265-282. Springer, Heidelberg (2007)

29. Groth, J., Ostrovsky, R., Sahai, A.: Non-interactive zaps and new techniques for NIZK. In: Dwork, C. (ed.) CRYPTO 2006. LNCS, vol. 4117, pp. 97-111. Springer, Heidelberg (2006)

30. Groth, J., Ostrovsky, R., Sahai, A.: Perfect non-interactive zero knowledge for NP. In: Vaudenay, S. (ed.) EUROCRYPT 2006. LNCS, vol. 4004, pp. 339-358. Springer, Heidelberg (2006)

31. Groth, J., Sahai, A.: Efficient non-interactive proof systems for bilinear groups. In: Smart, N.P. (ed.) EUROCRYPT 2008. LNCS, vol. 4965, pp. 415-432. Springer, Heidelberg (2008) 
32. Hazay, C., Katz, J., Koo, C.-Y., Lindell, Y.: Concurrently-secure blind signatures without random oracles or setup assumptions. In: Vadhan, S. (ed.) TCC 2007. LNCS, vol. 4392, pp. 323-341. Springer, Heidelberg (2007)

33. Hofheinz, D., Kiltz, E.: Secure hybrid encryption from weakened key encapsulation. In: Menezes, A. (ed.) CRYPTO 2007. LNCS, vol. 4622, pp. 553-571. Springer, Heidelberg (2007)

34. Juels, A., Luby, M., Ostrovsky, R.: Security of blind digital signatures. In: Kaliski Jr., B. (ed.) CRYPTO 1997. LNCS, vol. 1294, pp. 150-164. Springer, Heidelberg (1997)

35. Kiayias, A., Zhou, H.-S.: Concurrent blind signatures without random oracles. In: Yung, M. (ed.) SCN 2006. LNCS, vol. 4116, pp. 49-62. Springer, Heidelberg (2006)

36. Lysyanskaya, A., Ramzan, Z.: Group blind digital signatures: A scalable solution to electronic cash. In: Hirschfeld, R. (ed.) FC 1998. LNCS, vol. 1465, pp. 184-197. Springer, Heidelberg (1998)

37. Lysyanskaya, A., Rivest, R., Sahai, A., Wolf, S.: Pseudonym systems. In: Heys, H., Adams, C. (eds.) SAC 1999. LNCS, vol. 1758, pp. 184-199. Springer, Heidelberg (2000)

38. Martinet, G., Poupard, G., Sola, P.: Cryptanalysis of a partially blind signature scheme, or How to make $\$ 100$ bills with $\$ 1$ and $\$ 2$ ones. In: Crescenzo, G.D., Rubin, A. (eds.) FC 2006. LNCS, vol. 4107, pp. 171-176. Springer, Heidelberg (2006)

39. Meiklejohn, S., Shacham, H., Freeman, D.M.: Limitations on transformations from composite-order to prime-order groups: the case of round-optimal blind signatures. Cryptology ePrint Archive, Report 2010/474 (2010), http://eprint.iacr.org/2010/474

40. Okamoto, T.: Efficient blind and partially blind signatures without random oracles. Cryptology ePrint Archive, Report 2006/102 (2006), http://eprint.iacr.org/

41. Okamoto, T.: Efficient blind and partially blind signatures without random oracles. In: Halevi, S., Rabin, T. (eds.) TCC 2006. LNCS, vol. 3876, pp. 80-99. Springer, Heidelberg (2006)

42. Paillier, P.: Public-key cryptosystems based on composite degree residuosity classes. In: Stern, J. (ed.) EUROCRYPT 1999. LNCS, vol. 1592, pp. 223-238. Springer, Heidelberg (1999)

43. Paterson, K., Schuldt, J.: Efficient identity-based signatures secure in the standard model. In: Batten, L.M., Safavi-Naini, R. (eds.) ACISP 2006. LNCS, vol. 4058, pp. 207-222. Springer, Heidelberg (2006)

44. Pointcheval, D., Stern, J.: Security arguments for digital signatures and blind signatures. J. Cryptology 13(3), 361-396 (2000)

45. Shacham, H.: A Cramer-Shoup encryption scheme from the linear assumption and from progressively weaker linear variants. Cryptology ePrint Archive, Report 2007/074 (2007), http://eprint.iacr.org/

46. Waters, B.: Efficient identity-based encryption without random oracles. In: Cramer, R. (ed.) EUROCRYPT 2005. LNCS, vol. 3494, pp. 114-127. Springer, Heidelberg (2005)

47. Zhang, F., Kim, K.: ID-based blind signature and ring signature from pairings. In: Zheng, Y. (ed.) ASIACRYPT 2002. LNCS, vol. 2501, pp. 533-547. Springer, Heidelberg (2002) 